\title{
How much have the perinatal outcomes of triplet pregnancies improved over the last two decades?
}

\author{
Kyu-Sang Kyeong ${ }^{1}$, Jae-Yoon Shim², Soo-young $\mathrm{Oh}^{3}$, Hye-Sung Won ${ }^{2}$, Pil Ryang Lee ${ }^{2}$, Ahm Kim²,
} Sung-Cheol Yun ${ }^{4}$, Pureun-Narae Kang ${ }^{3}$, Suk-Joo Choi ${ }^{3}$, Cheong-Rae Roh ${ }^{3}$

\begin{abstract}
Department of Obstetrics and Gynecology, ${ }^{1}$ Hallym University Dongtan Sacred Heart Hospital, Hwaseong, ${ }^{2}$ Asan Medical Center, University of Ulsan College of Medicine, ${ }^{3}$ Samsung Medical Center, Sungkyunkwan University School of Medicine, Seoul; ${ }^{4}$ Department of Biostatistics, Asan Medical

Center, University of Ulsan College of Medicine, Seoul, Korea
\end{abstract}

\section{Objective}

This study was conducted to demonstrate the temporal trends in perinatal outcomes of triplet pregnancies over the last two decades.

\section{Methods}

The medical records of patients with triplet pregnancies at two Korean tertiary-care hospitals from 1992 to 2012 were retrospectively reviewed in regard to maternal and neonatal outcomes. The study was divided into two periods for analysis: period I (1992-2001) and period II (2003-2012).

\section{Results}

Over a 21-year period, 65 women with triplet pregnancies and 185 neonates were analyzed. Period II, when compared with period I, was associated with improved maternal outcomes, characterized by a decreased incidence of preeclampsia $(31.8 \%$ vs. $2.3 \%, P=0.002)$ and anemia $(68.2 \%$ vs. $30.2 \%, P=0.003)$ during pregnancy. Regarding neonatal aspects, the composite morbidity of period II was significantly decreased compared with that of period I, as assessed with a generalized estimating equation for logistic regression $(26.2 \%$ vs. $8.1 \%, P=0.03)$. Multivariable analysis revealed that the gestational age at delivery and the period were significantly associated with the composite neonatal morbidity $(P<0.001$ and 0.007 , respectively).

Conclusion

Improved neonatal morbidity was associated with a higher gestational age at delivery and with the more recent decade.

Keywords: Triplets; Pregnancy; Preterm birth; Pregnancy outcome

Received: 2018.09.10. Revised: 2018.12.06. Accepted: 2018.12.09. Corresponding author: Jae-Yoon Shim

Department of Obstetrics and Gynecology, Asan Medical Center, University of Ulsan College of Medicine, 88 Olympic-ro 43-gil, Songpa-gu, Seoul 05505, Korea

E-mail: jyshim@amc.seoul.kr

https://orcid.org/0000-0003-1881-8436
Corresponding author: Soo-young Oh

Department of Obstetrics and Gynecology, Samsung Medical Center, Sungkyunkwan University School of Medicine, 81 Ilwonro, Gangnam-gu, Seoul 06351, Korea E-mail: ohsymd@skku.edu https://orcid.org/0000-0003-3002-0048
Articles published in Obstet Gynecol Sci are open-access, distributed under the terms of the Creative Commons Attribution Non-Commercial License (http://creativecommons. org/licenses/by-nc/3.0/) which permits unrestricted non-commercial use, distribution, and reproduction in any medium, provided the original work is properly cited.

Copyright @ 2019 Korean Society of Obstetrics and Gynecology 


\section{Obstetrics \& Gynecology Science}

Kyu-Sang Kyeong, et al. Perinatal outcomes of triplet pregnancy

\section{Introduction}

The worldwide incidence of triplet pregnancies has increased considerably over the last 30 years $[1,2]$. There is no doubt that this increase in triplet births has been the result of a growing utilization of assisted reproductive technology (ART) and an increasing tendency for women to delay childbearing $[2,3]$. Although the birth rate has been decreasing annually in Korea, the rate of multiple pregnancy has increased with the advancement of ART [4-6]. Recent data in Korea have indicated a current multiple pregnancy rate of 32.9 per 1,000 live births in 2013 [7]. As in many other industrialized nations, the birth rate of triplets and higher-order multiples in Korea has exhibited a four-fold increase from 0.15 per 1,000 live births in 2003 to 0.59 per 1,000 live births in 2013 [7].

Triplet pregnancies are associated with increased risks of maternal complications, such as pregnancy-associated hypertension and gestational diabetes mellitus (GDM), and neonatal complications, which are mostly associated with preterm births $[8,9]$. Efforts are being made to reduce the complications associated with preterm births. Advances in perinatal care, including tocolytics, antenatal corticosteroids, and postnatal surfactant administration, have decreased the rate of neonatal morbidity for preterm births [10]. Some studies have shown that a complete course of an antenatal corticosteroid is associated with decreased risks of respiratory distress syndrome (RDS) and severe intraventricular hemorrhage (IVH) in triplet pregnancies [11,12]. New tocolytics, such as atosiban, have also been added to traditional betamimetics and magnesium sulfate. However, only a few studies have addressed changes in the perinatal outcomes of triplet pregnancies, and these studies have shown inconsistent results [13-15].

In ART pregnancies, selective fetal reduction (SFR) can be considered in some triplet pregnancies to improve neonatal outcomes by reducing the rates of early preterm births $[16,17]$. Considering the medical and ethical issues of SFR, the importance of providing accurate information on the most recent outcomes of triplet pregnancies cannot be overestimated. In this context, we considered it necessary to compare previous and the most recent outcomes of triplet pregnancies in Korea and to analyze the associated factors.

\section{Materials and methods}

This study retrospectively reviewed a total of 65 consecutive triplet pregnancies delivered at the two largest tertiarycare hospitals in Korea: the Asan Medical Center (AMC) and the Samsung Medical Center (SMC), from January 1992 to December 2012, with each center providing Institutional Review Board approval (approval number: 20160024).

Women who delivered before 20 weeks of gestation or those with the death of one or more of the fetuses before 20 weeks, were excluded from the cohort of triplet pregnancies. Neonates with reduced viability who were born before 23 weeks, those with major congenital anomalies, and those who experienced in utero fetal death were also excluded from the analysis of neonatal morbidity.

The admission, discharge, and outpatient records of pregnant women and neonates were evaluated. All information was reviewed by two of the authors. This 21-year study was divided into two periods: 1992 to 2001 (period I) and 2003 to 2012 (period II). There were no triplet births at either hospital in 2002. We evaluated the temporal trends in the maternal and neonatal outcomes of triplet pregnancies.

We followed the management guidelines presented by the American College of Obstetricians and Gynecologists for all triplets $[18,19]$. Management policies for triplet pregnancies have varied over time, and hospitalization for bed rest, prophylactic cerclage, and prophylactic tocolytics have not been generally proposed. Tocolytics were administered if clinically indicated and included one or more of the following: ritodrine, magnesium sulfate $\left(\mathrm{MgSO}_{4}\right)$, nifedipine, or atosiban. Betamimetics or $\mathrm{MgSO}_{4}$ were used as first-line agents. A dose of corticosteroids was administered between 23 and 34 weeks of gestation only in expectant mothers in whom preterm birth was highly probable. The delivery was mainly by elective cesarean section after 34 weeks.

We evaluated the maternal age, the gestational age at delivery, chorionicity, pregnancy complications of preterm labor (PTL), preterm premature rupture of membranes (PPROM), preeclampsia, GDM, the use of antenatal corticosteroids and tocolytics, and anemia during pregnancy. PTL was defined as a uterine activity causing progressive cervical changes at $<37$ weeks of gestation. Preeclampsia was diagnosed when hypertension at $\geq 140 / 90 \mathrm{mmHg}$ appeared after 20 weeks of gestation and was accompanied by proteinuria (defined as urinary excretion of $\geq 300 \mathrm{mg}$ of protein in 24 hours or a 


\section{Obstetrics \& Gynecology Science}

Vol. 62, No. 4, 2019

urine dipstick value of $1+$ or more). Screening for GDM in all triplet pregnancies was carried out between 24 and 28 weeks of gestation by measuring plasma glucose levels with the $50 \mathrm{~g}$ oral glucose tolerance test. A 1-hour post screening value of $>140 \mathrm{mg} / \mathrm{dL}$ was considered positive. A diagnosis of GDM was based on the recommendations of the fourth International Work Shop Conference on Gestational Diabetes, which utilizes the Carpenter-Coustan criteria [20]. Anemia during pregnancy was defined as hemoglobin concentrations from just before delivery: $<10.5 \mathrm{~g} / \mathrm{dL}$ in the second trimester of pregnancy or $<11.0 \mathrm{~g} / \mathrm{dL}$ in the third trimester [21].

The neonatal data included birth weight, length of hospitalization, Apgar scores, and the presence of RDS, bronchopulmonary dysplasia (BPD), retinopathy of prematurity (ROP), neonatal sepsis, severe IVH, and necrotizing enterocolitis (NEC). RDS was diagnosed based on the combination of clinical (tachypnea, grunting respirations, costal retraction, and cyanosis despite $40 \%$ oxygen therapy) and radiological (a diffuse reticulogranular pattern with air bronchogram) features [22]. BPD was defined as a condition requiring treatment with oxygen after 28 days or continuous oxygen dependence at a corrected gestational age of 36 weeks [23]. Grade III-IV IVH was considered as severe IVH. Grade III involved IVH occupying more than $50 \%$ of the ventricular area, and grade IV involved periventricular hemorrhagic infarction of adjacent white matter [24]. ROP was diagnosed according to the guidelines of the International Committee for the Classification of ROP [25]. NEC was diagnosed based on clinical symptoms and radiographic pneumatosis intestinalis based on Bell's staging criteria [26]. Both suspected sepsis and proven sepsis were included. Suspected sepsis was diagnosed when the blood test findings, such as the total leukocyte count, immature total neutrophils, platelet count, and C-reactive protein were abnormal and accompanied by clinical features of sepsis without identified bacteria. Proven sepsis was diagnosed with identified bacteria. The composite neonatal morbidity was defined as any of the four chronic neonatal morbidities: BPD, severe IVH, NEC, and ROP. Neonatal and infant mortality were excluded from the composite outcomes because all of the mortality cases had more than one morbidity factor.

For the descriptive statistical analysis, categorical variables are presented as numbers (percentages) and were compared using the $\chi^{2}$ test and Fisher's exact test. Continuous variables are expressed as the mean and were compared using the Student's unpaired t-test or the Mann-Whitney $U$ test. Differences between the two period groups (1992 to 2001 and 2003 to 2012) regarding composite neonatal morbidity were assessed with a generalized estimating equation (GEE) logistic regression, which accounts for the cluster effects. Variables with $P$-values $\leq 0.2$ in the univariate analyses were included in a multivariable GEE logistic regression model. The final model for the composite neonatal morbidity was determined by backward stepwise elimination procedures. A value of $P<0.05$ was interpreted as statistically significant. SAS software, version 9.1 (SAS Institute Inc., Cary, NC, USA), was used for statistical analyses.

\section{Results}

Over the 21-year study period, a total of 65 women delivered triplets ( 48 women at AMC and 17 women at SMC). Overall, the mean maternal age was 31.7 years, and 51 (78.5\%) of the 65 women were nulliparous. Regarding the conception method, $50(76.9 \%)$ triplet pregnancies were conceived through ART. The majority of the triplet pregnancies (95.4\%) were delivered by cesarean section. Three vaginal deliveries occurred: one due to delivery at 21 weeks of gestation, one due to the death of one fetus, and one due to precipitous labor. Chorionicity was ascertained for $80 \%$ of the triplets. Of the triplets, $56.9 \%(n=37)$ were trichorionic triamniotic, $16.9 \%(n=11)$ were dichorionic triamniotic, $6.1 \%(n=4)$ were monochorionic (3 triamniotic and 1 monoamniotic) and 20\% $(n=13)$ were of unknown chorionicity. Additionally, there were no complications resulting from the monochorionic pairs. Six cervical cerclages were performed, and indications of cervical cerclage were: a short cervical length (4 cases), an incompetent cervix ( 1 case), and a previous history of an incompetent cervix ( 1 case).

As depicted in Fig. 1, 10 out of 195 fetuses were excluded from the analysis of neonatal morbidity for the following reasons: $3(1.5 \%)$ due to a previable gestational age (at 21 weeks of gestation); $3(1.5 \%)$ due to intrauterine death (at 26,27 , and 28 weeks of gestation); and 4 (2.1\%) due to severe congenital anomalies (two with pulmonary atresia and two with severe aortic stenosis).

Period I (1992-2001) and period II (2003-2012) comprised 22 and 43 pregnant women with triplet pregnancies, respectively. Table 1 presents the maternal characteristics of the 


\section{Obstetrics \& Gynecology Science}

Kyu-Sang Kyeong, et al. Perinatal outcomes of triplet pregnancy

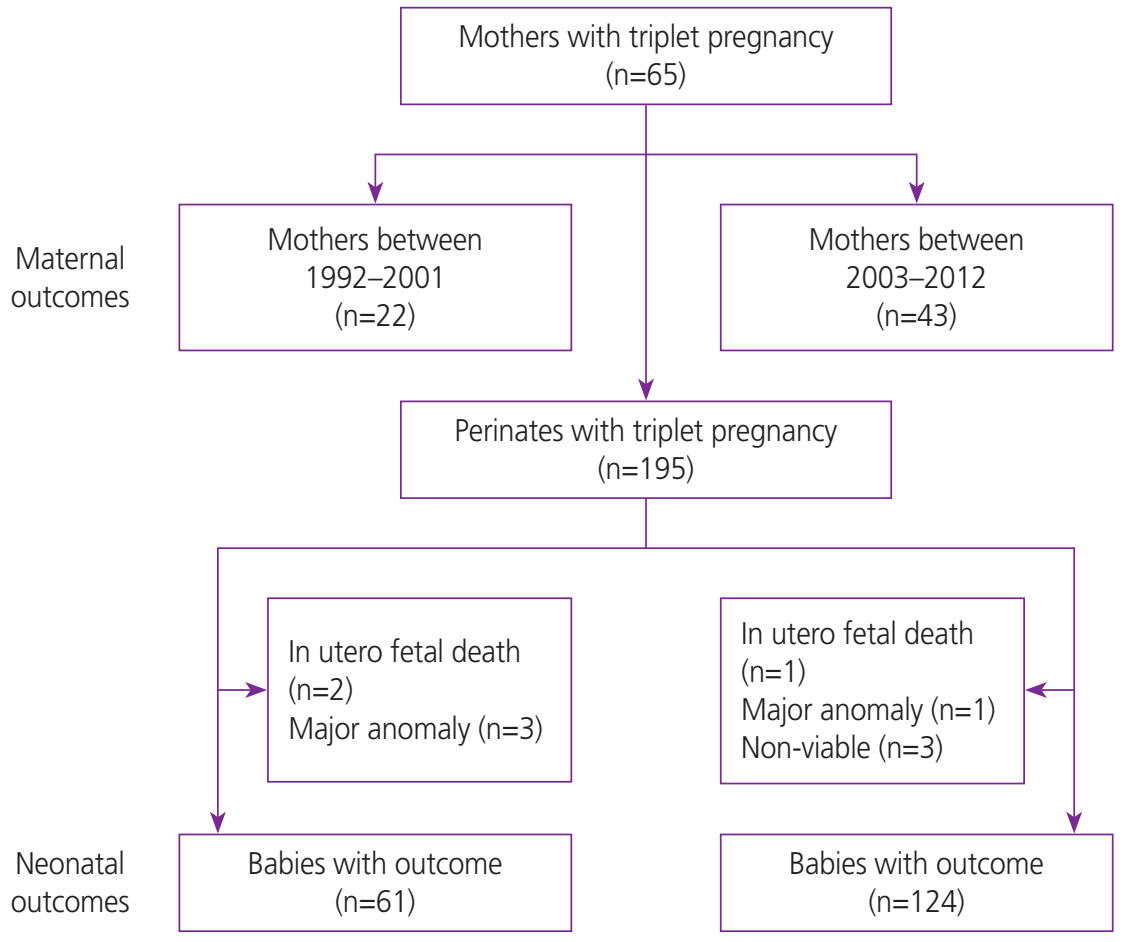

Fig. 1. Study participants and exclusion criteria.

Table 1. Maternal characteristics and outcomes of triplet pregnancies within two time periods

\begin{tabular}{|c|c|c|c|}
\hline Characteristics & $\begin{array}{l}\text { Period I (1992-2001) } \\
\text { ( } n=22)\end{array}$ & $\begin{array}{l}\text { Period II (2003-2012) } \\
\qquad(n=43)\end{array}$ & $P$-value ${ }^{\text {a) }}$ \\
\hline Maternal age (yr) & $31.4 \pm 4.2$ & $31.9 \pm 4.0$ & 0.629 \\
\hline Nulliparity & $17(77.3)$ & $34(79.1)$ & 0.868 \\
\hline ART & $18(81.8)$ & $32(74.4)$ & 0.757 \\
\hline Gestational age at delivery (wk) & $32.1 \pm 2.3$ & $32.2 \pm 3.7$ & 0.925 \\
\hline Delivery <28 wk & $0(0)$ & $5(11.6)$ & 0.158 \\
\hline Delivery $>34$ wk & $4(18.1)$ & $18(41.9)$ & 0.056 \\
\hline Trichorionic $^{\text {b) }}$ & $6 / 10(60)$ & $31 / 42(73.8)$ & 0.448 \\
\hline Antenatal corticosteroid & $4(18.2)$ & $11(25.6)$ & 0.367 \\
\hline Use of tocolytics & $10(45.5)$ & $21(48.8)$ & 0.796 \\
\hline Cesarean delivery & $21(95.5)$ & $41(95.3)$ & 0.985 \\
\hline PTL & $7(31.8)$ & $19(44.2)$ & 0.335 \\
\hline PPROM & $6(27.3)$ & $6(14.0)$ & 0.190 \\
\hline Preeclampsia & $7(31.8)$ & $1(2.3)$ & 0.002 \\
\hline GDM & $0(0)$ & $2(4.7)$ & 0.545 \\
\hline Cerclage operation & $1(4.5)$ & $5(11.6)$ & 0.655 \\
\hline Hemoglobin before delivery (g/dL) & $10.5 \pm 1.7$ & $11.5 \pm 1.7$ & 0.015 \\
\hline Anemia & $15(68.2)$ & $13(30.2)$ & 0.003 \\
\hline
\end{tabular}

Data presented as the mean \pm standard deviation or number (percentage).

ART, assisted reproductive technology; PTL, preterm labor; PPROM, preterm premature rupture of membranes; GDM, gestational diabetes mellitus.

${ }^{a}$ Categorical variables: the $\chi^{2}$ test or Fisher's exact test; Continuous variables: Student's unpaired $t$-test or the Mann-Whitney $U$ test; $P<0.05$ : statistically significant; ${ }^{b / W e}$ excluded triplets with unknown chorionicity (period I: $n=12$ and period II: $n=1$ ). 


\section{Obstetrics \& Gynecology Science}

Vol. 62, No. 4, 2019

study population for both periods. Maternal age, gestational age at delivery, chorionicity, the use of tocolytics, and the use of antenatal corticosteroids were not different between periods. Notably, the incidence of preeclampsia was markedly decreased in period II compared with period I (31.8\% vs. $2.3 \%, P=0.002$ ). As shown in Table 1, the incidence of anemia during pregnancy was significantly decreased in period II compared with period $\mathrm{I}$.

Table 2 summarizes the neonatal outcomes of the study population between periods I and II when analyzed with GEE logistic regression. Gestational age at delivery was not different between periods. When subdivided by gestational age at delivery as presented, there was a significant difference in each proportion between periods. The incidence of delivery under 28 weeks of gestational age was decreased, and the incidence of delivery over 34 weeks of gestational age was increased in period II compared with period I. The mean birth weight of the neonates tended to be higher in period II, but it did not reach statistical significance. Although the frequency of NICU admission did not significantly differ between periods, period II was associated with shorter hospital stays ( 30 vs. 18 days, $P=0.001$ ) and a lower incidence of Apgar scores below 7 at 5 minutes ( $23.0 \%$ vs. $5.6 \%, P<0.001)$. Although individual neonatal morbidity was not significantly different between periods, the composite neonatal morbidity was significantly decreased in period II compared with period I $(26.2 \%$ vs. $8.1 \%, P=0.033)$ with neonatal and infant mortality rates of triplet neonates in periods I and II being $0 \%$ vs. $0.8 \%$ and $0 \%$ vs. $2.4 \%$, respectively.

Table 3 shows maternal and neonatal characteristics of the study population according to the presence of composite neonatal morbidity, using a univariate GEE logistic regression. In this univariate analysis, the gestational age at delivery, PPROM, the neonatal hospital stay, birthweight, a 1-minute

Table 2. Neonatal outcomes of triplet pregnancies within two time periods

\begin{tabular}{lccc}
\hline Characteristics & $\begin{array}{c}\text { Period I (1992-2001) } \\
(\mathbf{n = 6 1 )}\end{array}$ & $\begin{array}{c}\text { Period II (2003-2012) } \\
(\mathbf{n = 1 2 4 )}\end{array}$ & $\boldsymbol{P}_{\text {-value }} \mathbf{a}^{\text {a) }}$ \\
\hline Gestational age at delivery (wk) & $32.3 \pm 2.3$ & $32.5 \pm 3.3$ & 0.704 \\
Delivery <28 wk & 0 & $12(9.7)$ & 0.009 \\
Delivery $>34$ wk & $12(19.7)$ & $53(42.7)$ & 0.002 \\
Birth weight (g) & $1,574 \pm 407$ & $1,683 \pm 465$ & 0.103 \\
1-min Apgar score $<4$ & $10(16.4)$ & $9(7.3)$ & 0.054 \\
5-min Apgar score $<7$ & $14(23.0)$ & $7(5.6)$ & $<0.001$ \\
NICU admission & $51(83.6)$ & $103(83.1)$ & 0.926 \\
Hospital stay (day) & $30(5-114)$ & $18(1-345)$ & 0.001 \\
Mechanical ventilation & $28(45.9)$ & $40(32.3)$ & 0.070 \\
Use of surfactant & $20(32.8)$ & $27(21.8)$ & 0.106 \\
Required phototherapy & $50(82.0)$ & $89(71.8)$ & 0.132 \\
RDS & $22(36.1)$ & $27(21.8)$ & 0.151 \\
BPD & $7(11.5)$ & $8(6.5)$ & 0.451 \\
ROP & $7(11.5)$ & $9(7.3)$ & 0.508 \\
Sepsis & $12(19.7)$ & $13(10.5)$ & 0.186 \\
Severe IVH & $5(8.2)$ & $4(3.2)$ & 0.267 \\
NEC & $4(6.6)$ & $2(1.6)$ & 0.090 \\
Composite morbidity & $16(26.2)$ & $10(8.1)$ & 0.033 \\
Neonatal mortality & $0(0)$ & $1(0.8)$ & 1.000 \\
\hline
\end{tabular}

Data are presented as the mean \pm standard deviation, median (range), or number (percentage).

NICU, neonatal intensive care unit; RDS, respiratory distress syndrome; BPD, bronchopulmonary dysplasia; ROP, retinopathy of prematurity; IVH, intraventricular hemorrhage; NEC, necrotizing enterocolitis.

${ }^{a)}$ Categorical variables: the $\chi^{2}$ test or Fisher's exact test; Continuous variables: Student's unpaired $t$-test or the Mann-Whitney $U$ test; $P<0.05$ : statistically significant. 


\section{Obstetrics \& Gynecology Science}

Kyu-Sang Kyeong, et al. Perinatal outcomes of triplet pregnancy

Apgar score $<4$, a 5-minute Apgar score $<7$, and the period, were found to be significantly related to the composite neonatal morbidity. When we performed multivariable GEE logistic regression with confounding factors and included factors with a $P$-value of $<0.2$, the gestational age at delivery and the period showed significant associations with the composite neonatal morbidity ( $P<0.001$ and $P=0.0074$, respectively). Period II demonstrated significantly decreased risk of composite neonatal morbidity compared with period I (adjusted odds ratio, $0.310 ; 95 \%$ confidence interval, 0.020-0.593).

\section{Discussion}

Our study demonstrated that the outcomes of triplet pregnancies significantly improved when period I (1992-2001) and period II (2003-2012) were compared, and these improvements were characterized by decreased composite neonatal morbidity during period II compared with period I. A pregnant woman with a triplet pregnancy has an increased risk of multiple neonatal morbidities. Therefore, we analyzed the characteristics of neonatal morbidities using a GEE logistic regression. Our multivariable analysis showed that independent contributing factors for the improvement of composite neonatal morbidity were attributable to a higher gestational age at delivery and the most recent period.

We have also shown that a higher gestational age at delivery, rather than an increase in the use of antenatal corticosteroids, contributed to the improvement in neonatal outcomes. There was no difference in neonatal gestational age at delivery between the two periods. However, deliveries under 28 and over 34 weeks of gestational age increased significantly in period II. The increase in deliveries under 28 weeks of gestational age can be explained by the lower threshold of viability. Instead of terminating the triplet pregnancies under 28 weeks of gestation, the patient was transferred to a tertiary hospital. In Korea, the rates of delivery prior to the 27th week of gestational age for multiple pregnancies in 1995, 2003 and 2012 have been reported to be $3(0.03 \%)$, $68(0.69 \%)$ and $358(2.29 \%)$, respectively [7]. However, we could not explain the increase in deliveries over 34 weeks of gestational age. Atosiban has been used in Korea since 2006 and was used in this study. However, noting an increased birth rate after 34 weeks of gestation in maternal characteristics ( $P=0.056)$ resulting from the use of tocolytics was difficult because betamimetics and $\mathrm{MgSO}_{4}$ are used as first-line

Table 3. Univariate and multivariable generalized estimating equation logistic regression analysis for composite neonatal morbidity

\begin{tabular}{|c|c|c|c|c|c|c|}
\hline \multirow{2}{*}{ Characteristics } & \multicolumn{3}{|c|}{ Univariable analysis } & \multicolumn{3}{|c|}{ Multivariable analysis } \\
\hline & OR & $95 \% \mathrm{Cl}$ & $P$-value ${ }^{\text {a) }}$ & A-OR & $95 \% \mathrm{Cl}$ & $P$-value ${ }^{\text {a) }}$ \\
\hline Maternal age (yr) & 0.985 & $0.810-1.198$ & 0.882 & & & \\
\hline Gestational age at delivery (wk) & 0.568 & $0.465-0.693$ & $<0.001$ & 0.433 & $0.303-0.620$ & $<0.001$ \\
\hline Nulliparity & 0.579 & $0.102-3.302$ & 0.538 & & & \\
\hline Cesarean delivery & 0.231 & $0.013-3.962$ & 0.312 & & & \\
\hline ART & 1.727 & $0.303-9.851$ & 0.538 & & & \\
\hline PTL & 1.033 & $0.306-3.485$ & 0.958 & & & \\
\hline PPROM & 4.107 & $1.064-15.853$ & 0.040 & & & \\
\hline Preeclampsia & 3.107 & $0.594-16.248$ & 0.179 & & & \\
\hline Antenatal corticosteroid & 1.173 & $0.267-5.156$ & 0.833 & & & \\
\hline Neonatal hospital stay (day) & 80.014 & $13.963-458.505$ & $<0.001$ & & & \\
\hline Birth weight & 0.995 & $0.994-0.997$ & $<0.001$ & & & \\
\hline 1-min Apgar score $<4$ & 13.842 & $4.244-45.149$ & $<0.001$ & & & \\
\hline 5-min Apgar score $<7$ & 10.927 & $3.307-36.104$ & 0.001 & & & \\
\hline Period II & 0.247 & $0.068-0.894$ & 0.033 & 0.310 & $0.020-0.593$ & 0.007 \\
\hline
\end{tabular}

OR, odds ratio; A-OR, adjusted odds ratio; $\mathrm{Cl}$, confidence interval; ART, assisted reproductive technology; PTL, preterm labor; PPROM, preterm premature rupture of membranes.

${ }^{\text {a) } A s s e s s e d ~ w i t h ~ a ~ g e n e r a l i z e d ~ e s t i m a t i n g ~ e q u a t i o n ~ l o g i s t i c ~ r e g r e s s i o n . ~} P<0.05$ : statistically significant. 


\section{Obstetrics \& Gynecology Science}

Vol. 62, No. 4, 2019

drugs [27] and because there was no difference in the use of the various tocolytics between the two periods. When our period II data is compared to U.S. birth data, gestational age at delivery (32.5 vs. 31.97 weeks) and mean birth weight $(1,683$ vs. $1,660 \mathrm{~g})$ were similar. Our period II data also had a lower rate of delivery under 32 weeks (30.2\% vs. 36.8\%) and a lower rate of birth weight of under $1,500 \mathrm{~g}(29.8 \%$ vs. $35 \%$ ) compared to U.S. birth data. We believe that this difference is probably due to an increased incidence of gestational age over 34 weeks.

In a similar study, despite the use of new tocolytics, such as oxytocin receptor antagonists or calcium channel blockers, no prolonged pregnancies or delayed preterm deliveries of triplets occurred. Furthermore, the proportion of newborns with extreme prematurity ( $<28$ weeks) has not changed over the last three decades [14]. Ballabh et al. [15], analyzed the neonatal outcomes of triplets from 1993 to 2000. An increase in the use of antenatal corticosteroids was observed over that study period, but the incidence of RDS remained fairly constant. There was no definitive trend in the frequencies of surfactant use, patent ductus arteriosus, IVH, or ROP [15].

In our study, we found that the use of antenatal corticosteroids was similar between periods. The use of antenatal corticosteroids in singleton pregnancies with a risk of preterm birth lowers the incidence and severity of RDS [28]. Additionally, according to a systemic review, antenatal corticosteroid therapy enhances the circulatory stability of preterm neonates, lowering the risks of IVH, NEC, and systemic infection during the first 48 hours of life [29]. Although some studies have reported that a complete course of antenatal corticosteroid therapy significantly lowers the rates of RDS and IVH in preterm neonates from multiple pregnancies [11,30], many studies have not been able to identify the source of the improvement in neonatal outcomes $[14,15]$. Whether the effect of corticosteroid therapy decreases in multiple gestations has not been determined. However, Jobe [31] has hypothesized that the reason why the effect of antenatal corticosteroid therapy is low in twins is not because of the standard dose, but because the effect of corticosteroid therapy varies depending on the reaction of the fetus. Jobe [31] insists that in the PTL of twin pregnancies, there is not enough stress for the fetus to react to antenatal glucocorticoids.

Preeclampsia is related to the patient's socioeconomic status [32]. The decreased incidence of preeclampsia in Ko- rea can be related to large improvements in socioeconomic status and the introduction of the universal voucher scheme $[33,34]$. However, the incidence of preeclampsia during period II was extremely low. A possible explanation for this extremely low incidence may be the influence of the small sample size. During the study period, there was a significant increase in the antepartum hemoglobin level, whereas the frequency of women who developed anemia declined. This is presumably attributed to education regarding the optimal amount of iron supplementation for women with triplet pregnancies in prenatal care and to improved nutrition [35].

SFR in triplet pregnancies has been performed to decrease pregnancy loss, antenatal complications, birth before 36 weeks, and neonatal death [17]. However, a meta-analysis of 893 triplets reported that although SFR decreased the early preterm birth rate, SFR increased the miscarriage rate before 24 weeks from $4.4 \%$ to $8.1 \%$ [16]. Regardless of the change in the miscarriage rate, the meaning of a miscarriage after SFR differs from that of a spontaneous miscarriage. Moreover, if parents with a history of infertility undergo an abortion, a miscarriage may cause psychological trauma to the parents and is also a burden for the doctor who performs the procedure. Although the neonatal outcome and prognosis of a triplet pregnancy are poorer than those of singleton and twin pregnancies, advances in perinatal and neonatal care have changed the outcomes of triplet pregnancies over the last few decades [14]. However, a recent study reported that neonatal and infant mortality of triplets have not changed in Korea between 2009 and 2015 [36]. Further research regarding improvements in triplet pregnancy outcomes would be useful for providing counseling regarding SFR to women with triplet pregnancies and a history of infertility.

Although this study included two tertiary-care hospitals in Korea, the relatively small sample size of the study population may limit the power of some of the results derived from this study. To overcome this disadvantage, we performed a multivariable analysis to uncover the independent factors associated with improved neonatal outcomes in the most recent period and found that the significant independent predictors for neonatal morbidity were lower gestational age at delivery and the period (early). In addition, another limitation of the study was that the long-term outcomes of the neonates were not included.

In summary, the composite neonatal outcome of triplet pregnancies was significantly improved during the last two 


\section{Obstetrics \& Gynecology Science}

Kyu-Sang Kyeong, et al. Perinatal outcomes of triplet pregnancy

decades and was associated with a higher gestational age at delivery in the most recent period. This new data may provide very useful information for physicians and women with high-order multiple gestations, especially when compared with data from earlier time periods.

\section{Conflict of interest}

No potential conflict of interest relevant to this article was reported.

\section{Ethical approval}

The study was approved by the Institutional Review Board of Asan Medical Center (IRB No. 2016-0024) and performed in accordance with the principles of the Declaration of Helsinki.

\section{References}

1. Martin JA, Hamilton BE, Ventura SJ, Osterman MJ, Kirmeyer S, Mathews TJ, et al. Births: final data for 2009. Natl Vital Stat Rep 2011;60:1-70.

2. Black M, Bhattacharya S. Epidemiology of multiple pregnancy and the effect of assisted conception. Semin Fetal Neonatal Med 2010;15:306-12.

3. Ferraretti AP, Goossens V, de Mouzon J, Bhattacharya S, Castilla JA, Korsak V, et al. Assisted reproductive technology in Europe, 2008: results generated from European registers by ESHRE. Hum Reprod 2012;27:2571-84.

4. Hur YM, Kwon JS. Changes in twinning rates in South Korea: 1981-2002. Twin Res Hum Genet 2005;8:76-9.

5. Choi YM, Chun SS, Han HD, Hwang JH, Hwang KJ, Kang IS, et al. Current status of assisted reproductive technology in Korea, 2009. Obstet Gynecol Sci 2013;56:353-61.

6. Lee YJ, Kim MN, Kim YM, Sung JH, Choi SJ, Oh SY, et al. Perinatal outcome of twin pregnancies according to maternal age. Obstet Gynecol Sci 2019;62:93-102.

7. Yoon YO. Births statics: final data for 2013. Daejeon: Statistics Korea; 2014 [cited 2018 Oct 20]. Available from: http://kostat.go.kr.

8. Luke B, Brown MB. Maternal morbidity and infant death in twin vs triplet and quadruplet pregnancies. Am J Obstet Gynecol 2008;198:401.e1-10.

9. The ESHRE Capri Workshop Group. Multiple gestation pregnancy. Hum Reprod 2000;15:1856-64.

10. Fanaroff AA, Stoll BJ, Wright LL, Carlo WA, Ehrenkranz RA, Stark $A R$, et al. Trends in neonatal morbidity and mortality for very low birthweight infants. Am J Obstet Gynecol 2007;196:147.e1-8.

11. Blickstein I, Shinwell ES, Lusky A, Reichman B; Israel Neonatal Network. Plurality-dependent risk of respiratory distress syndrome among very-low-birth-weight infants and antepartum corticosteroid treatment. Am J Obstet Gynecol 2005;192:360-4.

12. Blickstein I, Reichman B, Lusky A, Shinwell ES; Israel Neonatal Network. Plurality-dependent risk of severe intraventricular hemorrhage among very low birth weight infants and antepartum corticosteroid treatment. Am J Obstet Gynecol 2006;194:1329-33.

13. Skrablin S, Kuvacic I, Kalafatic D, Peter B, GvericAhmetasevic S, Letica-Protega N, et al. Perinatal care improves the outcome of triplets. Eur J Obstet Gynecol Reprod Biol 2002;104:26-31.

14. Weissman A, Ulanovsky I, Burke Y, Makhoul IR, Blazer S, Drugan A. Triplet pregnancies--a three-decade perspective: do we fare better? Eur J Obstet Gynecol Reprod Biol 2013;170:82-4.

15. Ballabh P, Kumari J, AlKouatly HB, Yih M, Arevalo R, Rosenwaks $Z$, et al. Neonatal outcome of triplet versus twin and singleton pregnancies: a matched case control study. Eur J Obstet Gynecol Reprod Biol 2003;107:2836.

16. Papageorghiou AT, Avgidou K, Bakoulas V, Sebire NJ, Nicolaides $\mathrm{KH}$. Risks of miscarriage and early preterm birth in trichorionic triplet pregnancies with embryo reduction versus expectant management: new data and systematic review. Hum Reprod 2006;21:1912-7.

17. Dodd JM, Crowther CA. Reduction of the number of fetuses for women with triplet and higher order multiple pregnancies. Cochrane Database Syst Rev 2003:CD003932.

18. American College of Obstetricians and Gynecologists. ACOG educational bulletin. Special problems of multiple gestation. Number 253, November 1998 (Replaces Number 131, August 1989). Int J Gynaecol Obstet 1999;64:323-33. 


\section{Obstetrics \& Gynecology Science}

Vol. 62, No. 4, 2019

19. American College of Obstetricians and Gynecologists Committee on Practice Bulletins-Obstetrics; Society for Maternal-Fetal Medicine; ACOG Joint Editorial Committee. ACOG Practice Bulletin \#56: Multiple gestation: complicated twin, triplet, and high-order multifetal pregnancy. Obstet Gynecol 2004;104:869-83.

20. Metzger BE, Coustan DR; The Organizing Committee. Summary and recommendations of the Fourth International Workshop-Conference on Gestational Diabetes Mellitus. Diabetes Care 1998;21 Suppl 2:B161-7.

21. Centers for Disease Control and Prevention. Recommendations to prevent and control iron deficiency in the United States. MMWR Recomm Rep 1998;47:1-29.

22. Stahlman M, Hedvall G, Dolanski E, Faxelius G, Burko H, Kirk V. A six-year follow-up of clinical hyaline membrane disease. Pediatr Clin North Am 1973;20:433-46.

23. Jobe AH, Bancalari E. Bronchopulmonary dysplasia. Am J Respir Crit Care Med 2001;163:1723-9.

24. Volpe JJ. Neurology of the newborn. 4th ed. Philadelphia (PA): W.B. Saunders; 2001.

25. International Committee for the Classification of Retinopathy of Prematurity. The International Classification of Retinopathy of Prematurity revisited. Arch Ophthalmol 2005;123:991-9.

26. Neu J. Necrotizing enterocolitis: the search for a unifying pathogenic theory leading to prevention. Pediatr Clin North Am 1996;43:409-32.

27. Hwang HS, Na SH, Hur SE, Lee SA, Lee KA, Cho GJ, et al. Practice patterns in the management of threatened preterm labor in Korea: a multicenter retrospective study. Obstet Gynecol Sci 2015;58:203-9.

28. Liggins GC, Howie RN. A controlled trial of antepartum glucocorticoid treatment for prevention of the respiratory distress syndrome in premature infants. Pediatrics 1972;50:515-25.

29. Roberts D, Dalziel S. Antenatal corticosteroids for accelerating fetal lung maturation for women at risk of preterm birth. Cochrane Database Syst Rev 2006:CD004454.

30. Kuk JY, An JJ, Cha HH, Choi SJ, Vargas JE, Oh SY, et al. Optimal time interval between a single course of antenatal corticosteroids and delivery for reduction of respiratory distress syndrome in preterm twins. Am J Obstet Gynecol 2013;209:256.e1-7.

31. Jobe AH. Antenatal steroids in twins. Am J Obstet Gynecol 2003;188:856.

32. Silva LM, Coolman M, Steegers EA, Jaddoe VW, Moll HA, Hofman A, et al. Low socioeconomic status is a risk factor for preeclampsia: the Generation R Study. J Hypertens 2008;26:1200-8.

33. Choe SA, Min HS, Cho SI. Decreased risk of preeclampsia after the introduction of universal voucher scheme for antenatal care and birth services in the Republic of Korea. Matern Child Health J 2017;21:222-7.

34. Choe SA, Min HS, Cho SI. The income-based disparities in preeclampsia and postpartum hemorrhage: a study of the Korean National Health Insurance cohort data from 2002 to 2013. Springerplus 2016;5:895.

35. Goodnight W, Newman R; Society of Maternal-Fetal Medicine. Optimal nutrition for improved twin pregnancy outcome. Obstet Gynecol 2009;114:1121-34.

36. Ko HS, Wie JH, Choi SK, Park IY, Park YG, Shin JC. Multiple birth rates of Korea and fetal/neonatal/infant mortality in multiple gestation. PLoS One 2018;13:e0202318. 